\title{
Management of the first newborn delivered by a mother with COVID-19 in South Korea
}

\begin{abstract}
Key message
Question: How to manage the newborn born to the coronavirus disease 2019 (COVID-19) pregnant?

Finding: Medical staff managed the delivery and neonatal care of a COVID-19 pregnant patient was based on the "Guidelines for COVID-19 response."

Meaning: We desire that our management will help treat for subsequent patients and there should be updated continuously the prevention and control consensus strategies for newborn COVID-19.
\end{abstract}

On March 6, 2020, a female neonate was born to a 28-yearold woman in Daegu Fatima Hospital at 37 weeks and 6 days. On February 24, twelve days before the delivery, her mother was confirmed with coronavirus disease 2019 (COVID-19) and stayed self-isolated at home. Her father, who is also a member of the Shincheonji religious organization, was diagnosed with COVID-19 2 days before his wife. We report a first case of infant born to mother with confirmed COVID-19 in South Korea and describe the management approach for neonates born to women with confirmed COVID-19 including practical method for prevention and control infection.

\section{What happened in Daegu in February 2020?}

On February 18, 2020, the 31st confirmed patient of COVID19 was reported in Daegu, South Korea, as a member of the Shincheonji religious organization. ${ }^{1)}$ It caused the huge cluster of infection and triggered the outbreak of COVID-19 infection. As characteristic of religion which young female believers take up the majority, cases of COVID-19 pregnant and infant patient are expected to increase. At the beginning of the outbreak of COVID-19, the Public Relief Hospitals were not operated. And the general delivery hospitals were unable to proceed with the treatment and delivery of pregnant women suspected with COVID-19. On February 23, 2020, Daegu Fatima Hospital was designated to hospital by Daegu city government to manage confirmed or suspected pregnant patients with COVID-19.

\section{How to delivery of a COVID-19 pregnant patient?}

Approach for delivery and neonatal care of a COVID-19 pregnant patient was based on the Guidelines for COVID-19 response by Korea Centers for Disease Control and Prevention (KCDC) and The Korean Society of Pediatric Infectious Diseases. ${ }^{2)}$

\section{What was the preparedness of the medical team for de- livery?}

Because of self-isolation at home, COVID-19 pregnant patient could not visit the hospital for prenatal evaluation. $\mathrm{Ob}$ stetrician and medical staff in Daegu Fatima Hospital checked and evaluated her clinical condition on the phone every day. She had a mild fever and coughing for 2 days during the early self-isolation period. But she showed no symptoms of dyspnea or tachypnea. After discussing with the patient, the obstetrician decided to perform caesarean section delivery on the day of admission. We asked the patient not to breast-feed or stay with her baby after delivery. The delivery room was rearranged into an operating room for the caesarean section of the patient, and remaining inpatients were discharged or transferred to other hospitals.

\section{How was the management of the delivery?}

As soon as the pregnant patient arrived at the hospital, she was guided to the isolation clinic by medical staff who wore personal protective equipment (PPE) and checked physical examination, laboratory tests, and chest x-rays were performed. She was moved to the delivery room through a separate passage using an independent elevator. All medical staff wore PPE including gown, gloves, medical mask, face shield for eye and face protection. Noticeably, the obstetrician used PPE with specific powered air purifying respirator for respiratory protection. All medical staff used vinyl-treated medical instruments and disposable kits in delivery room and medical instruments used in the operation were sent for disinfection. Wastes from operation room were disposed according to infectious medical waste protocol. . $^{3}$ 


\section{How to manage the newborn born to the COVID-19 pregnant?}

A female baby was born at 37 weeks and 6 days gestation with active crying and movement. After medical staff checked the Apgar score, the baby was transferred to a negative pressure isolation room in the neonatal intensive care unit (NICU) using a mobile incubator. Medical staff moved the incubator along a separate passage.

\section{Isolating \& postnatal care of the newborn}

The baby born to the patient was considered as a "suspected" case until the test results for COVID-19 were verified. She was admitted to the NICU and isolated in a negative pressure isolation room in which the exhaust is filtered through highefficiency particular air filters. The baby weighed 3,010 g and Apgar scores were 8 at 1 minute and 9 at 5 minutes. Her vital signs were stable and within the normal limit. Medical staff closely monitored her for symptoms related the COVID-19 infection symptoms. (e.g., fever, respiratory difficulty, and other respiratory symptoms). All medical staff wore PPE including gown, gloves, medical mask, face shield during managing the baby. A disposable baby bottle was used for the newborn, and used fabrics were disposed according to the infectious medical waste protocol.

\section{How to diagnose COVID-19 in newborn?}

1) Real-time reverse transcription polymerase chain reaction (RT-PCR) for COVID-19 assays.

The baby was examined for COVID-19 infection right after being admitted to the NICU. Medical staff collected specimens for of the upper respiratory tract (nasopharyngeal and oropharyngeal), gastric lavage fluid, blood, skin, urine, and stool right after she arrived at the NICU. And we also examined the specimens of the mother's placenta, cord blood, and amniotic fluid to confirm mother-to-newborn (vertical) transmission. Upper respiratory tract specimens were collected from both nasopharyngeal and oropharyngeal swabs and they were put in a same sample tube. Blood sample was collected and kept in a serum separator tube. Urine, stool, amniotic fluid, and cord blood specimens were collected and stored in sterile specimen containers at $4^{\circ} \mathrm{C}$ until ready for shipment to the KCDC. Upper respiratory tract specimens were tested with real-time reverse transcription polymerase chain reaction assays in Daegu Fatima Hospital and other specimens were sent to KCDC because of reagent problem. The serologic test and genetic sequencing for COVID-19 were not performed.

2) Medical staff did laboratory tests of the baby such as complete blood count, C-reactive protein (CRP), and chest x-ray. Blood test results showed a white blood cell count of 24,000/ $\mu \mathrm{L}$, a hemoglobin concentration of $17.4 \mathrm{~g} / \mathrm{dL}$, a platelet count of $280,000 / \mathrm{mL}$, and a CRP level of $0.01 \mathrm{mg} / \mathrm{dL}$. Chest x-rays showed normal findings.
3) Results of RT-PCR assays (1st) were negative in all of the 10 specimens (upper respiratory tract, gastric lavage fluid, blood, skin, urine, stool, placenta, cord blood, and amniotic fluid). And second test for RT-PCR was conducted after 48 hours from birth by nasopharyngeal and oropharyngeal swab. Second sample for RT-PCR results was also negative.

\section{How did the baby's hospitalization changed?}

As the second test for detection of COVID-19 was confirmed negative, the newborn was released from isolation. However, she was kept away from her mother who remained in isolation. As the newborn was in good health, medical staff decided to discharge her and return her to her guardians.

\section{What should we think of more?}

As of March 15,2020, more than 8,000 confirmed cases with COVID-19 were reported and over 70\% of them came from Daegu and Gyeongsangbuk-do areas. ${ }^{4)}$ Because of the distinct characteristics of their religion, many young women attended Shincheonji church. Therefore, the number of pregnant women and newborns infected with COVID-19 was expected to increase. Actually, a recent report showed predominance by the age group of the 20s and females may have caused by the outbreak related to a religious group in Daegu. ${ }^{5)}$

We could not certain whether mothers with COVID-19 could transmit the virus through breast milk. KCDC recommend infants should breastfed carefully even if they are confirmed or suspected with COVID-19. If a mother is confirmed as negative for COVID-19, her newborn should be fed with expressed breast milk of a healthy mother.

Data on coronavirus infections in newborns are limited. And vertical transmission of COVID-19 was not certain whether it is possible or not. Karimi-Zarchi et al. $\left.{ }^{6}\right)$ reported that there is no evidence for vertical transmission of COVID-19 between neonates and their mothers with COVID-19 in late pregnancy. But recently Lamouroux et al. ${ }^{7)}$ reported there was the possibility of per-partum infection of COVID-19 in infected neonates whom symptoms appeared within 2-4 days of life, since the virus could not be isolated from vaginal swabs. In our case, we confirmed there was no evidence about intrauterine transmission of COVID-19 and pregnancy complications due to COVID-19 infection. Since our results are based on only 1 case, the vertical transmission of COVID-19 cannot be completely ruled out.

We report a first case of infant born to mother with confirmed COVID-19 in South Korea and describe the preparatory process for delivery, management of the newborn infant. COVID-19 infection is continuing. We desire that our management will help treat for subsequent patients and there should be updated continuously the prevention and control consensus strategies for newborn COVID-19. 


\section{Conflicts of interest}

No potential conflict of interest relevant to this article was reported.

\section{Acknowledgments}

Korea Center for Disease Control and prevention and The Korean Society of Pediatric Infectious Disease provided guidelines for examination and safe delivery

See the commentary "What are considerations for neonates at risk for COVID-19?" via https://doi.org/10.3345/cep. 2020.01074 .

Eun-Kyung Lee, $\mathrm{MD}^{1}$, Won Duck Kim, $\mathrm{MD}^{1}$, Dong Won Lee, $\mathrm{MD}, \mathrm{PhD}^{1}$, Sang-Ah Lee, $\mathrm{MD}^{2}$

${ }^{1}$ Department of Pediatrics, Daegu Fatima Hospital, Daegu, Korea

${ }^{2}$ Department of Infectious Internal Medicine, Daegu Fatima Hospital, Daegu, Korea

Corresponding author: Eun-Kyung Lee, MD. Department of Pediatrics, Daegu Fatima Hospital, 99 Ayang-Ro, Dong-gu, Daegu 41199, Korea

凶E-mail: bbowwow@daum.net, https://orcid.org/0000-0002$8878-5265$

\section{References}

1. Korea Centres for Disease Control and prevention. The updates on COVID-19 in Republic of Korea, as of 18 February, 2020. Cheongju (Korea): Korea Centers for Disease Control and Prevention, 2020.

2. Kim KH, Cho EY, Kim DH, Kim HW, Park JY, Eun BW, et al. Guidelines for coronavirus disease 2019 response in children and adolescents. Pediatr Infect Vaccine 2020;27:24-34.

3. Korea Centres for Disease Control and prevention. Guidelines for waste safty management for COVID-19 response. Cheongju (Korea): Korea Centers for Disease Control and Prevention, 2020.

4. Korea Centres for Disease Control and prevention. The updates on COVID-19 in Republic of Korea, as of 24 March, 2020. Cheongju (Korea): Korea Centers for Disease Control and Prevention, 2020.

5. Korean Society of Infectious Diseases, Korean Society of Pediatric Infectious Diseases, Korean Society of Epidemiology, Korean Society for Antimicrobial Therapy, Korean Society for Healthcare-associated Infection Control and Prevention, Korea Centers for Disease Control and Prevention. Report on the Epidemiological Features of Coronavirus Disease 2019 (COVID-19) Outbreak in the Republic of Korea From January 19 to March 2, 2020. J Korean Med Sci 2020;35:e112.

6. Karimi-Zarchi M, Neamatzadeh H, Dastgheib SA, Abbasi H, Mirjalili SR, Behforouz A, et al. Vertical transmission of coronavirus disease 19 (COVID-19) from infected pregnant mothers to neonates: a review. Fetal Pediatr Pathol. 2020;39:246-50.

7. Lamouroux A, Attie-Bitach T, Martinovic J, Leruez-Ville M, Ville Y. Evidence for and against vertical transmission for severe acute respiratory syndrome coronavirus 2. Am J Obstet Gynecol 2020;223:91.e1-91.e4. 